\title{
Predicting Chemical Potential and Osmotic Pressure of Polysaccharide Solutions by Molecular Simulations
}

\author{
Jörg Sauter and Andrea Grafmüller* \\ Theory and Bio-Systems, Max Planck Institute of Colloids and Interfaces, Potsdam, \\ Germany \\ E-mail: andrea.grafmueller@mpikg.mpg.de
}

\section{Method Details}

\subsection{Noble Water Method}

The chemical potential can be approximated by using ${ }^{1}$

$$
\begin{aligned}
\mu & \simeq-k_{B} T \ln \left(\frac{V / \Lambda^{d}}{N+1}\right)-k_{B} T \ln \int\left\langle\exp \left[\frac{-\Delta U^{(N+1, N)}}{k_{B} T}\right]\right\rangle_{N} \mathrm{~d} s_{N+1} \\
& =\mu^{\text {ideal }}+\mu^{\text {ex }}
\end{aligned}
$$

where $\Delta U^{(N+1, N)}=U\left(s^{N+1}\right)-U\left(s^{N}\right)$ denotes the potential energy difference for the insertion of a single particle, $k_{B}$ is Boltzmann's constant, and $\Lambda^{d}$ the thermal wavelength in $d$ dimensions. Here, $T$ denotes the temperature, $V$ denotes the volume, and $N$ the number of particles.

${ }^{*}$ To whom correspondence should be addressed 
In this approximation, the chemical potential is split into an ideal gas part $\mu^{\text {ideal }}$ and an excess part $\mu^{\mathrm{ex}}$ containing the configurational contribution. With $N_{w}$ water and $N_{p}$ solute molecules the chemical potential of water is

$$
\mu_{w}=\left(\frac{\partial G}{\partial N_{w}}\right)_{T, P, N_{p}}
$$

where $G$ is the Gibb's free energy. The ideal part in (1) can be calculated analytically and the excess part of the chemical potential $\mu_{w}^{\mathrm{ex}}$ is equivalent to the hydration free energy of water $\Delta G$ (or $\Delta A$ in an appropriate NVT ensemble) obtained from vapor pressure experiments and simulations. ${ }^{2,3}$

The second term in equation (1) offers a way to calculate $\mu_{w}^{\text {ex }}$ by performing $n$ random water insertions into a pre-calculated $N$-particle trajectory by

$$
\mu_{w}^{\mathrm{ex}} \simeq-k_{B} T \ln \frac{1}{n} \sum_{j=1}^{n}\left\langle\exp \left[\frac{-\Delta U^{\left.\left(N_{w+1}, N_{w}\right)\right)}}{k_{B} T}\right]\right\rangle_{N}
$$

using brute force sampling and assuming ergodicity. This is the TPI method.

In addition to massive convergence problems for complex systems, the insertions are performed uniformly and thus an energy bias is introduced which results in systematic errors in the approximation of $\mu_{w}^{\mathrm{ex}}$. Especially for solute-solvent systems, that have a higher density than pure water, uniform insertions are likely to result in insertions very close to other particles and representing a state that is very unlikely in a true ensemble. For close contacts the repulsive term of the Lennard-Jones potential is the determining factor, therefore in this case, $\Delta U^{\left(N_{w+1}, N_{w}\right)}$ will be very large and $\mu_{w}^{\mathrm{ex}}$ is expected to be higher than the true value. Thus, the direct use of the method should be avoided for dense systems.

The noble water Method introduced by Knopp et. al ${ }^{4}$ aims to circumvent this problem. The method combines TPI with alchemical mutations of the water molecules to a less dense, so called noble water, to reduce the bias of the TPI method which is applied after the mutation. The free energy difference $\Delta A_{B A R}^{\mathrm{ex}}$ for this transformation is calculated per water 
molecule using either TI as in the original method or BAR, as done here.

We mutate from regular water to noble water because the phase space overlap, measured in terms of relative entropy,$^{5}$ is better than for the mutation from noble water to regular water. For the mutation, the following $26 \lambda$ values are used to transform the LJ and electrostatic interactions:

vdw- $\lambda$ values $=\begin{array}{lllllllllllllllll}0.0 & 0.05 & 0.1 & 0.15 & 0.175 & 0.2 & 0.25 & 0.3 & 0.35 & 0.4 & 0.45 & 0.5 & 0.55 & 0.6 & 0.65 & 0.7 & 0.75\end{array}$ 0.80 .850 .8750 .90 .91250 .9250 .950 .9751 .0

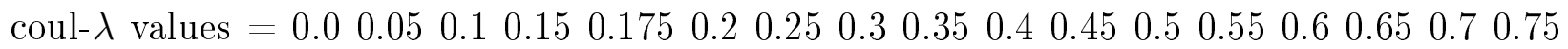

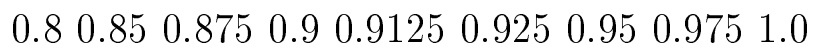

The system was sampled for 2 ns and the first 100 ps were neglected as additional equilibration. To ensure convergence, the evolution of $\Delta A_{B A R}^{\mathrm{ex}}$ as a function of simulation time was evaluated. Convergence was archived within 100 ps (200 ps total simulation time) for the pure water systems.

For the TPI method we used a 50 ns trajectory with a frame interval of 2 ps and performed 2 million insertions per frame. To check for convergence, $\mu_{T P I}^{\mathrm{ex}}$ was monitored as a function of simulation time. Only minor fluctuation in $\mu_{T P I}^{\mathrm{ex}}$ were observed after $10 \mathrm{~ns}$ for all water models. Using twice the amount of insertions and a doubled simulation time gave virtually indistinguishable results, i.e. a deviation smaller than $0.001 \mathrm{~kJ} \mathrm{~mol}^{-1}$.

\subsection{Direct Bennets Acceptance Ratio}

As for TPI, the principle of free energy methods such as BAR is to insert a molecule into

a given system. Instead of using a pre-calculated trajectory, this is accomplished using 27 $\lambda$ states for which the system is sampled and allowed to equilibrate. The following lambda values were used:

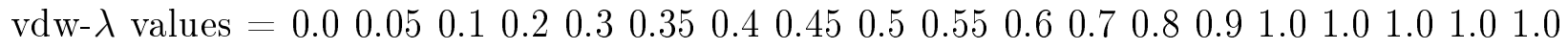
1.01 .01 .01 .01 .01 .01 .01 .0

coul- $\lambda$ values $=0.00 .00 .00 .00 .00 .0 \quad 0.0 \quad 0.00 .00 .00 .00 .00 .00 .00 .00 .10 .20 .30 .40 .50 .6$ 
0.70 .80 .90 .950 .9751 .0

Only minor fluctuations were observed after 100 ps (200 ps total simulation time), therefore, we assume convergence.

\subsection{Osmotic Pressure Method}

The bulk density of TIP5P water in a pure water system at a pressure of 1 bar is found to be $980.252 \mathrm{~kg} / \mathrm{m}^{3}$. The osmotic pressure systems are set up to match this value of the bulk density, taking into account the excluded volume effects of the repulsive walls at the box boundaries in the z-direction.

To test how much the results are affected by the small size of the bulk water region, a test simulation with a significantly larger bulk water region of $6 \mathrm{~nm}$ water on each side was performed for a solution with a $1 \mathrm{~m}$ glucose concentration, using $200 \mathrm{~ns}$ of simulation time.

The osmotic pressure value found in this simulation is $2.57 \pm 0.097 \mathrm{MPa}$. The value found for the $2 \mathrm{~nm}$ water bath at the same glucose concentration is $2.51 \pm 0.01 \mathrm{MPa}$. These values are in good agreement within the error margins. The larger error in the $6 \mathrm{~nm}$ bath system is due to the shorter simulation time.

\subsection{Metadynamics Calculations}

To obtain the free energy maps we used the Plumed $2.2 .2^{6}$ implementation of metadynamics ${ }^{7}$ with GROMACS 5.1.2. We simulated a $\beta$-D-glc(1-4) $\beta$-D-glc (cellobiose) molecule hydrated with 1904 water molecules for 100ns using the Leap-Frog integrator with a 2 fs time-step size and the temperature of $298.15 \mathrm{~K}$ was controlled using the Nosé-Hoover thermostat. Electrostatic interactions were treated with the Particle Mesh Ewald method. ${ }^{8} \mathrm{~A} 1.4 \mathrm{~nm}$ cut-off was used. The bias was added every picosecond to the $\Phi$ (O5,C1,O4,C4) and $\Psi$ (C1,O4,C4,C3) dihedral angles and used a height of $0.01 \mathrm{~kJ} \mathrm{~mol}^{-1}$ and a variance of $\sigma=0.2$. 


\section{Chemical Potential in Saccharide Solutions}

The noble water method was originally devised for the calculation of the excess chemical potential of water in dense polymer structures. Here, we applied the method to $\beta-D$-glucose solutions of different concentrations from $1 \mathrm{~m}$ to $5.5 \mathrm{~m}$. The results for the GLYCAM06 TIP5P FF differ by an order of magnitude from the experimental value.

Plotting the BAR contribution for converting water molecules to noble water over simulation time reveals that, in contrast to the pure water systems, convergence is not achieved within 2 ns nor within an extended simulation time of 10 ns, as shown in Figure I for a $\beta-D$-glucose solution at $4 \mathrm{~m}$ concentration. This leads to large errors. Analysis of the

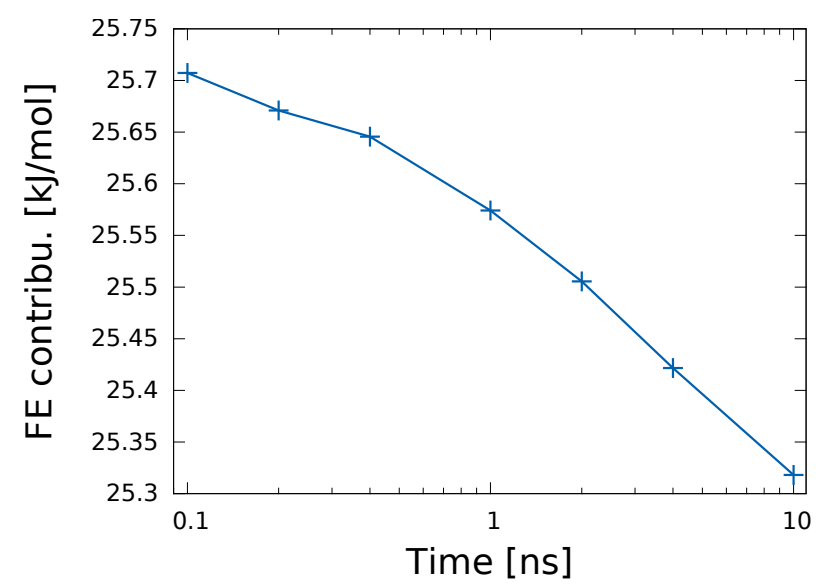

Figure I: The free energy contribution $\Delta A_{B A R}^{\mathrm{ex}}$ to the excess chemical potential $\mu_{w}^{\mathrm{ex}}$ is plotted versus the simulation time. The plot shows no convergence up to $10 \mathrm{~ns}$.

simulation trajectories shows that in the noble water method the saccharides aggregate due to the reduced sugar-water interactions, which disturbs the fragile balance between the interactions. The saccharide glasses formed as a result and the associated sampling problems are likely to be the main reason for the failure of the results to converge. Besides these sampling problems, the magnitude of the TPI error for pure water already exceeds the precision required to observe the solute effects on the chemical potential. The experimental chemical potential difference between pure water and a $4 \mathrm{~m} \beta-D$-glucose solution is only approximately $-0.2 \mathrm{~kJ} \mathrm{~mol}^{-1}$ at $298.15 \mathrm{~K}$, whereas the TPI bias for most water models was 
$>1 \mathrm{~kJ} \mathrm{~mol}^{-1}$. Taking into account all the above factors, we can conclude that the noble water method is not suitable to predict the excess chemical potential of saccharide solutions with sufficient accuracy.

\section{References}

(1) Frenkel, D.; Smit, B. Understanding molecular simulation: from algorithms to applications; Elsevier (formerly published by Academic Press), 2002; Vol. 1; pp 1-638.

(2) Ben-Naim, A.; Marcus, Y. J. Chem. Phys. 1984, 81, 2016-2027.

(3) Jorgensen, W. L.; Blake, J. F.; Buckner, J. K. Chem. Phys. 1989, 129, 193-200.

(4) Knopp, B.; Suter, U. W.; Gusev, A. A. Macromolecules 1997, 30, 6107-6113.

(5) Wu, D.; Kofke, D. A. J. Chem. Phys 2005, 123, 054103.

(6) Tribello, G. A.; Bonomi, M.; Branduardi, D.; Camilloni, C.; Bussi, G. Comput. Phys. Commun. 2014, 185, 604-613.

(7) Laio, A.; Parrinello, M. Proc. Natl. Acad. Sci. 2002, 99, 12562-12566.

(8) Darden, T.; York, D.; Pedersen, L. J. Chem. Phys. 1993, 98, 10089-10092. 